In Crescendo, 2019; 10(4): 593-610

Fecha de recepción: 01/07/2019

Fecha de aceptación: 23/12/2019

\title{
FACTORES DE RIESGO QUE GENERAN LA AGRESIVIDAD EN LOS NIÑOS Y NIÑAS DENTRO DEL CONTEXTO ESCOLAR DE LA INSTITUCIÓN EDUCATIVA ANORÍ, Y EL CENTRO EDUCATIVO RURAL LA HERMOSA DURANTE EL AÑO 2018, DEL MUNICIPIO DE ANORÍ
}

\author{
RISK FACTORS THAT GENERATE AGGRESSIVENESS IN BOYS AND GIRLS \\ WITHIN THE SCHOOL CONTEXT OF THE EDUCATIONAL INSTITUTION \\ ANORÍ, AND THE RURAL EDUCATIONAL CENTER LA HERMOSA \\ DURING THE YEAR 2018, OF THE MUNICIPALITY OF ANORÍ
}

Leidy M. Flórez', Beatriz E. Agudelo ${ }^{2}$, Santos B. Rengifo ${ }^{3}$

\section{RESUMEN}

El entorno escolar tiene una influencia decisiva en el comportamiento de los niños, niñas y jóvenes pues genera en ellos un sentimiento de competitividad y superioridad dentro del grupo. Acciones que recurren en actitudes agresivas que se presentan en eventos que desencadena en la expresión de superioridad o poder frente a los demás. Pues ciertas condiciones proporcionan al niño señales de que su conducta agresiva puede tener consecuencias gratificantes. Los resultados de esta investigación confirman que la violencia escolar es el producto de una compleja combinación de variables, entre las que debe considerarse el entorno escolar, el entorno familiar y el entorno social. Los resultados encontrados deben conducir hacia una

1 Especialista Administración de Informática Educativa docente de la institución Anorí correo lemaflome@quepasa.com

2 Especialista Administración de Informática Educativa correo abee1967@hotmail.com

3 Magister en Gestión de la Tecnología Educativa. docente de la Institución Educativa Manuel José Sierra del Municipio de Girardota - Antioquia - Colombia correo: berosa05@gmail.com 
reflexión sobre los valores y conductas que desde los procesos de socialización trasmiten a nuestros estudiantes desde la Institución y el centro educativo rural del Municipio de Anorí. En consecuencia, y puesto que las experiencias escolares son parte significativa del proceso estudiantil donde se aprenden de los otros, es necesario intervenir sobre aquello que representa una influencia negativa para el grupo, sobre todo si se tiene en cuenta que los problemas de violencia entre pares pueden ser la antesala de otras conductas problemáticas posteriores: abuso sexual, violencia en las relaciones de pareja o delincuencia.

PALABRAS CLAVE: Quechua, Millennials, Idioma, Lengua y Cultura.

\section{ABSTRACT}

The school environment has a decisive influence on the behavior of children and young people because it generates a feeling of competitiveness and superiority within the group. Actions that recur in aggressive attitudes that occur in events that trigger the expression of superiority or power in front of others. For certain conditions give the child signs that his aggressive behavior can have gratifying consequences. The results of this research confirm that school violence is the product of a complex combination of variables, among which the school environment, the family environment and the social environment must be considered. The results found should lead to a reflection on the values and behaviors that from the processes of socialization transmit to our students from the Institution and the rural educational center of the Municipality of Anorí. Consequently, and since school experiences are a significant part of the student process where they learn from others. It is necessary to intervene on what represents a negative influence for the group, especially if we take into account that the problems of violence between peers can be the prelude to other subsequent problematic behaviors: sexual abuse, violence in relationships or delinquency.

KEY WORDS: School violence, aggression, sexual abuse and physical abuse.

\section{INTRODUCCIÓN}

Anorí es un municipio ubicado en el departamento de Antioquia - Colombia en el cual existen dos Instituciones Educativas y cincuenta y siete centros educativos rurales, los cuales son los encargados de impartir la educación a los niños y niñas que tienen a su cargo, con el propósito de mejorar la calidad educativa. Para el desarrollo del proyecto se tomará como punto de referencia la institución educativa Anorí y el centro educativo rural La Hermosa, este último desarrolla la metodología escuela nueva.

El modelo pedagógico Escuela nueva, fue diseñado en Colombia a mediados de los años setenta por Vicky Colbert, Beryl Levinger y Óscar Mogollón, donde buscaban el mejoramiento de la calidad educativa de la básica primaria del país. 
Dewey citado por (Gadotti, 2002) la define como

Un conjunto de doctrinas y principios tendientes a revisar, por un lado, los fundamentos de la finalidad de la educación, por el otro, las bases de aplicación de la ciencia a la técnica educativa. Tales tendencias nacieron de nuevas necesidades sentidas por el hombre, en el cambio de civilización en que nos encontramos, y son más evidentes bajo ciertos aspectos, en los países que más sufrieron, directa o indirectamente, los efectos de la conflagración europea. (Gadotti, 2002)

El modelo en Colombia se desarrolla actualmente en centros educativos rurales, ubicados en los lugares más apartados del territorio colombiano, conocido como las zonas rurales (veredas) donde es más común la aplicabilidad de la metodología, manejada por uno o dos docentes, quienes atienden los grados de preescolar a quinto (multigrado) bajo las estrategias de guías de estudio, por grupo de estudiantes.

La Escuela Nueva se nutrió de la máxima representativa del aprender haciendo, donde se promueven un aprendizaje activo, participativo, cooperativo, dinámico, autónomo y colaborativo. Centró sus procesos educativos en los niños, a través de situaciones cotidianas que suceden en la familia, la historia de la vereda, de los productos agropecuarios que allí se producen y en el entorno inmediato del niño en general. Encaminados a responder a las características y demandas de los mismos (cada niño avanza de acuerdo a sus capacidades y disponibilidad de tiempo), todo en sana relación con la comunidad circundante y con el mundo actual.

En cada uno los centros educativos se recopiló información la cual será necesaria para el desarrollo efectivo del proyecto. Durante su desarrollo es importante tener claridad con el concepto de agresividad, además aquellos factores que intervienen en la agresión humana. Factores tales como la ira, la intolerancia, el maltrato físico, o psicológico, el entorno socio económico, la alimentación, los medios de comunicación, y la normatividad vigente en relación con la violencia escolar.

Estos factores influyen de una manera negativa con respecto a las relaciones interpersonales que crea el sujeto agresor con las personas más cercanas. En este sentido, Serrano, (s.f), expresa que la agresividad se presenta se forma física, verbal o desplazada, en esta última el niño arremete contra los objetos de la persona que ha dado origen del conflicto.

Se ha evidenciado diferentes comportamientos en los educandos que van en contra de los principios morales e institucionales y de su integridad personal, mediante diferentes eventos, entre los que se pueden mencionar: 
- El mal uso de sus materiales de estudio.

- El uso del pupitre como tablero de grafitis.

- El mal uso que hace de: puertas, lámparas, estantes, ventiladores, materiales de trabajo y materas.

- El uso de la sección de unidades de sanitario para dejar consignas del plantel.

- Los niños y niñas en su quehacer escolar, conviven con los trastornos agresivos que se manifiestan en forma de lenguaje obsceno, patadas, agresión física, manipulaciones, hacia otras personas u objetos que le rodeen.

El comportamiento agresivo complica las relaciones que los estudiantes establecen a lo largo de su vida escolar. Pues, la escuela tiende a discriminar estos niños, sometiéndolos a un rechazo y a un desequilibrio emocional que puede generar un aumento en las conductas agresivas. En algunas ocasiones, sus pares inconscientemente actúan de forma agresiva cuando sienten que algo o alguien amenazan su integridad. Igualmente, sucede en diferente escenario de su vida cotidiana -la familia, los vecinos o grupos de pares- en los que expresan diferentes episodios para llamar la atención o para ser tenido en cuenta en los procesos.

El ambiente dentro del cual el niño y la niña se desarrolla tiene una influencia, a veces decisiva sobre su propio comportamiento. En algunas familias se piensa que cuando un(a) niño(a) presenta conducta agresiva, es porque aprenden comportamientos inadecuados en la calle o en la escuela. Acciones que se deben analizar desde diferentes eventos: El comportamiento de los padres o cuidadores en su convivencia, la pérdida de un ser querido, el no cumplimiento de las reglas, una inadecuada alimentación y la forma cómo se castigan los niños entre otros.

Situación que afecta la posibilidad de mejorar la conducta agresiva de los infantes y que la mayoría de los casos es el reflejo de las conductas de los adultos y es aprendida por los niños a través de la observación, como lo determinan ciertos estudios, entre los que se pueden mencionar:

- Caracterización y análisis socio demográfico.

- Análisis de formas de castigo.

- Análisis de las manifestaciones de agresividad e influencia del contexto.

- Análisis del programa "Joven sano". 
Lo anterior conlleva a una interrogante ¿Cuáles son los factores de riesgo que generan la agresividad en los niños y niñas de la Institución educativa Anorí y del Centro Educativo Rural La Hermosa durante el año 2018, del Municipio de Anorí?

\section{Objetivo general}

Analizar los factores de riesgo que generan la agresividad en los niños y niñas dentro del contexto escolar de la Institución educativa Anorí, y el Centro Educativo Rural La Hermosa durante el año 2018, del Municipio de Anorí

\section{Objetivos específicos}

$\checkmark$ Identificar las diferentes situaciones que generan la agresividad en las niñas y los niños.

$\checkmark$ Señalar las características y patrones que influyen en los niños agresivos, en la dinámica escolar.

$\checkmark$ Deliberar acerca de la influencia del contexto socioeconómico en la conducta agresiva de los niños y niñas la Institución Educativa Anorí, y el Centro Educativo Rural La Hermosa durante el año 2018, del Municipio de Anorí.

$\checkmark$ Construir una presentación en power point con el fin de socializar el trabajo de investigación a docentes y entes involucrados.

\section{Justificación}

La agresividad en las niñas y niños dentro del contexto educativo en el ciclo de primaria de la Institución educativa Anorí y del Centro Educativo Rural La Hermosa del Municipio de Anorí, nace con la necesidad de conocer las situaciones que están influyendo para que este fenómeno sea común en nuestras instituciones y, por ende, en la sociedad, circunstancia que permitió cuestionarnos y plantear una propuesta con el fin de llegar a un acercamiento con la población vulnerable.

Para la difusión de sus resultados se utilizarán diferentes medios de comunicación, con el propósito que la comunidad educativa conozca la problemática y realice eventos positivos para superarla. Uno de ellos puede ser la elaboración de folletos informativos en los cuales se puedan visualizar las conclusiones y estrategias de prevención frente a los factores de riesgo, en este proceso es importante tener en cuenta a: 
Los discentes o alumnos, como agentes efectivos del proceso de enseñanzaaprendizaje y en quienes recae la atención y el interés en la enseñanza. A ellos con el propósito de que conozcan los factores de la agresividad y su incidencia en la realidad, para que reflexionen y en efecto, aquellos que ya hacen parte del problema comiencen a cambiar su postura.

Los docentes, o enseñantes encargados de facilitar el aprendizaje, para que el alumno los alcance de la mejor manera posible, además quienes se dedican profesionalmente a la enseñanza, bien sea con carácter general, o se hayan especializado en una determinada área del conocimiento, asignatura, disciplina académica, ciencia o arte o simplemente un docente en la zona rural o escuela unitaria. Para que tengan una herramienta guía que los ayude a conocer esas anomalías presentadas en el plantel en cuanto a la agresividad y puedan así direccionar el proceso y tomar las salidas pertinentes.

Los padres de familia, como los agentes que están más cerca del educando y quienes les ofrecen una estabilidad emocional, social y económica. En este ambiente el educando aprende tempranamente a dialogar, a escuchar, a conocer y desarrollar sus derechos y deberes como persona humana. Para que tenga en cuenta los resultados de la investigación y los factores que determinan la agresividad y puedan evitar que sus hijos caigan en este flagelo y los que están inmersos en el problema tomen los correctivos necesarios.

La institución, como espacio de interacción del educando, educador y de los padres de familia. Quienes a su vez conforman la comunidad educativa; es la más beneficiada de la investigación ya que, si los actores antes mencionados se apropian de dichos factores, comiencen a respirar un ambiente más sano, cordial y propicio para el aprendizaje, originando así una mejor vida social y por ende un progreso significativo en la valoración académica.

A las autoridades competentes, para que dentro del componente de promoción y prevención u otro que esté más relacionado, para que analicen los resultados, los evalúen y los puedan tener a su disponibilidad para cuando ellos lo crean necesario y pertinente, con la finalidad de mitigar el problema existente.

\section{MARCOTEÓRICO}

Con este marco teórico se busca apropiarse de una base conceptual que permita acercarnos a las concepciones relacionadas con los factores de riesgos que generan la agresividad en los niños en los planteles seleccionados. 
El problema de la agresividad infantil es uno de los trastornos que más afectan a padres y maestros. A menudo estos se enfrentan a niños agresivos, manipuladores o rebeldes pero ellos no saben muy bien cuáles son las causas y cómo deben actuar frente a estos niños, para llegar a modificar esta conducta. (Pearce, 1995) como se citó en Pérez (2009) la agresividad deriva del latín "agredí" que significa "atacar". Implica que una persona está decidida a imponer su voluntad a otra e incluso a causar daño físico o psíquico.

Desde la perspectiva psicoanalítica, la agresividad según Laplache y Pontalis, (1974), es definida como un conjunto de tendencias que se actualizan en conductas reales, dirigidas a dañar a otro o humillarlo, etc. Por su parte la agresión puede adoptar diferentes modalidades relacionadas con la acción violenta y destructiva. Por lo tanto, no hay conducta tanto negativa, ni positiva, ni simbólica efectivamente realizada, que no pueda funcionar como agresión.

De lo anterior se deduce que el comportamiento agresivo complica las relaciones dentro del ambiente escolar, es por ello que en los planteles educativos se adopta un reglamento interno llamado manual de convivencia (Comunidad Educativa 2001 cap. 6 y 7), instrumento y/o documento que contiene pautas establecidas con participación de los estamentos comunitarios con base en la democracia, tolerancia, derechos humanos. Basado en apartes de:

La Constitución Política de Colombia: Artículo 44, derechos fundamentales de los niño.

La ley 1098 del 8 de noviembre de 2006. Código de la infancia y de la adolescencia

Determina las pautas para regular el ejercicio de las libertades y derechos de los alumnos, así como el cumplimiento de los deberes en el interior de las instituciones educativas. El Decreto 1423 (1993)

Los cuales orientan en el buen funcionamiento de los diferentes roles involucrados en el proceso de enseñanza - aprendizaje.

Además, los comportamientos agresivos complican las relaciones que las personas establecen a lo largo de su vida, pues la sociedad tiende a aislar a estas personas sometiéndolas a un rechazo y a un desequilibrio emocional que puede generar un aumento en las conductas agresivas.

En algunas ocasiones, las personas inconscientemente actúan de forma agresiva cuando sienten que algo o alguien amenazan su integridad, así mismo, en situaciones de estrés, para llamar la atención de su familia, vecinos o grupos de pares. 
En los primeros años de la infancia, es normal que se presenten situaciones de agresividad, pero algunos niños persisten en su conducta agresiva y en su incapacidad para dominar su mal genio y en el momento de ingresar a la escuela es inevitable demostrar su comportamiento agresivo, dando lugar al rechazo de sus compañeros.

Existen muchos motivos por los que un niño puede tener comportamientos agresivos; es decir, comportamientos mediante los cuales demuestra una cierta hostilidad hacia los demás a través de conductas físicas agresivas o bien mediante un lenguaje (insultos, amenazas, etc.). Las personas con las que convive el niño o la niña y las experiencias que viven a lo largo de su existencia, modelarán de forma decisiva estos rasgos temperamentales iníciales. Por tanto, el ambiente dentro del cual el niño y la niña se desarrollan tiene una influencia, a veces decisiva, sobre su propio comportamiento.

En algunas familias se piensa que cuando un niño o niña presenta conducta agresiva, es porque aprenden comportamientos inadecuados en la calle o en la escuela, y en lugar de analizar las formas (la de los padres) de afrontar las dificultades que se dan en la convivencia (discusiones entre padres, ausencia de la figura paterna o materna, pérdida de un ser querido, no cumplimiento de una regla, inadecuada alimentación, entre otros.), aplican castigos a sus hijos, cerrando toda posibilidad de mejorar esta conducta agresiva que la mayoría de los casos es producida por ellos y aprendida por los niños a través de la observación, como lo determinan ciertos estudios.

Concluyendo, se puede respaldar que, cuando se habla de agresividad escolar no se puede dar como concepto aislado sino como una de las complicaciones del síndrome de déficit atencional e hiperactividad del escolar que lentamente y en forma gradual va perdiendo el autoestima y por ende, recurre a elementos de defensa y ataque para mostrar algún tipo de superioridad frente a sus pares e incluso frente a sus educadores. Es un síntoma de alerta para estudiar urgentemente, algún tipo de trastorno comicial de carácter parcial o trastornos del sueño del tipo comicial.

Si en una familia se dan continuos episodios de violencia, el niño al llegar al aula de clase trae consigo esta agresividad reinante en su hogar, haciendo lo mismo que copia allí con sus compañeros, útiles de trabajo, maestros y hasta consigo mismo; de la misma forma, el barrio donde vive el niño y el lugar donde está ubicada su escuela es determinante en la aparición de la conducta agresiva, tal 
como lo afirma Parra (1992) Si las instituciones están situadas en un lugar afectado por la violencia, muchos de los alumnos seguramente tratarán de conseguir armas o podrán ser víctimas inocentes de los eventos de violencia.

\section{METODOLOGÍA}

Para realizar la investigación se partirá del entorno educativo, familiar y social en el que viven niños y niñas que presentan marcados brotes de agresividad. En otras instancias se tendrá en cuenta las hipótesis planteadas alrededor de esta problemática que se evidencia en los educandos.

\section{Tipo de estudio}

Después de un análisis riguroso el presente proyecto está relacionado con:

El nivel: explicativo ya que se analizan las causas que generan la agresividad en los niños niñas.

El tipo de estudio es explicativo, se partirá de los eventos presentados por los niños y las niñas en relación con las expresiones de violencia dentro del contexto educativo y familiar.

La población involucrada en el proyecto corresponde a los estudiantes y docentes que conforman el ciclo de primaria que pertenecen a la Institución Educativa Anorí, con un total de 717 estudiantes de los cuales 343 son niñas, 352 niños y 23 docentes dirigidos por dos coordinadoras. Los educandos del Centro Educativo Rural La Hermosa conformada por 22 educandos, distribuidos en 12 mujeres, 10 hombres y un docente de aula. Para el desarrollo de la investigación se tomará como muestra el 10\% del total de niñas y niñas al igual del total de educadores, de los planteles educativos mencionados.

La muestra es un "subconjunto representativo de un universo o población." (Morlés, 1994, p. 54) para el proyecto será electiva, no al azar. Por ejemplo, las entrevistas serán realizadas a 10 docentes, 25 estudiantes distribuidos en los diferentes grados y planteles y 15 padres de familia; la selección se hará de los educandos que se presume están más relacionados con la problemática, y que en el seguimiento educativo se evidencien eventos de agresividad en el plantel, fuera de él, o en su defecto, los educandos líderes de los diferentes grados.

La investigación está conformada por dos variables, la primera, la independiente, la cual constituye al objeto de investigación, es decir las causas o condi- 
ciones del entorno de los niños y en segundo lugar la dependiente la agresividad y/o violencia en los niños y niñas.

\section{Métodos y técnicas de recolección de datos}

Para el desarrollo de este proyecto, en primera instancia, se tendrán en cuenta los objetivos, para luego investigar en los diferentes documentos que permitirán conocer más acerca del tema. Posteriormente, se desarrollará el trabajo de campo tomando como referencia a los niños y niñas y su cotidianidad. Se utilizará como instrumento la encuesta la cual será estructurada, como el mecanismo de interacción con los educandos y por ende con su contexto.

Toda la información recopilada será sistematizada y tabulada a través del programa Excel para luego ser tenida en cuenta en el proceso de análisis.

El instrumento utilizado para la recolección de los datos que potencializarán la investigación y que permitirá obtener información, será:

La encuesta, será aplicada a docentes, entorno familiar y estudiantes que se desempeñan en el ciclo de primaria de la Institución educativa Anorí y el Centro Educativo Rural La Hermosa.

\section{La encuesta}

La encuesta es el intercambio de ideas, significados y sentimientos sobre el mundo y los eventos, cuyo medio principal son las palabras. Es una interacción en la cual se exploran diferentes realidades y percepciones, donde el investigador intenta ver las situaciones de la forma cómo la ven sus informantes y comprender por qué se comportan de la manera que dicen hacerlo.

Se aplicará la encuesta tipo explicativa; la cual consiste en determinar las relaciones de causa y efecto, identificando cuál de esas causas se presenta con mayor frecuencia entre los alumnos estudiados.

Recolectada toda la información, se tabularán los resultados obtenidos de las entrevistas aplicadas, estos se registrarán en una ficha de las cuales obtendrán conclusiones, que posteriormente se verán reflejadas en acciones desde las dependencias que puedan involucrarse con la prevención a grupos vulnerables. 


\section{Tratamiento de la información}

\section{Análisis tabla causas}

Cuando los(as) adolescentes se comportan de manera agresiva, ia cuál de los siguientes factores lo atribuyes?

Tabla 1

\begin{tabular}{|c|c|c|c|c|c|c|c|c|c|c|}
\hline & Opciones & & $\begin{array}{l}\text { Ares c } \\
\text { amilia }\end{array}$ & & & cado & & & diant & \\
\hline a) & A que lo han aprendido de & Si & $\%$ & No & $\mathrm{Si}$ & $\%$ & No & $\mathrm{Si}$ & $\%$ & No \\
\hline & hermanos u otros) & 3 & $18 \%$ & 12 & 3 & $43 \%$ & 7 & 11 & $44 \%$ & 14 \\
\hline b) & $\begin{array}{l}\text { A que en su hogar se viven } \\
\text { situaciones conflictivas. }\end{array}$ & 4 & $16 \%$ & 11 & 2 & $20 \%$ & 8 & 6 & $24 \%$ & 19 \\
\hline c) & $\begin{array}{l}\text { A que la forma como los - } \\
\text { as han educado los lleva a } \\
\text { ser agresivos. }\end{array}$ & 2 & $8 \%$ & 13 & 0 & $0 \%$ & 0 & 3 & $12 \%$ & 22 \\
\hline d) & $\begin{array}{l}\text { A que no han recibido } \\
\text { afecto en su hogar. }\end{array}$ & 3 & $18 \%$ & 12 & 3 & $43 \%$ & 7 & 1 & $4 \%$ & 24 \\
\hline e) & $\begin{array}{l}\text { A que imitan modelos de } \\
\text { la televisión o de juegos } \\
\text { electrónicos. }\end{array}$ & 3 & $18 \%$ & 12 & 3 & $43 \%$ & 7 & 2 & $8 \%$ & 23 \\
\hline
\end{tabular}

Elaboración de los investigadores.

\section{Encuesta realizada a docentes}

Los docentes agentes dinamizadores del proceso de enseñanza y de aprendizaje quienes guían a los educando en su desarrollo y quienes tienen bajo su responsabilidad orientarlos en su formación; para el desarrollo de este proceso fueron vinculados para la entrevista diez (10) de ellos, los cuales se desempeñan en diferentes ambientes, unos urbanos y otros rurales.

En momentos de ocio, ellos se enfrentaron a una interrogante, en algunos seleccionaron solo una respuesta, pero en otros fueron varias las respuestas, que poco a poco avanza como material de insumo para el conocimiento de la agresividad y la forma como se evidencian los factores que la generan en los niños y niñas.

Después de tabuladas las entrevistas (ver tabla 2) se evidencia, la agresividad según los docentes:

Las respuestas denotan un señalamiento culpando a la sociedad, ya que esta ha permitido la conformación de pandillas o grupos que han llevado a los jóvenes a trasgredir normas en cualquiera de sus contextos. 
Es una constante en los hogares de los estudiantes las familias disfuncionales, a esto se le suma la carencia de pautas de crianza reflejándose en la pérdida de valores, que a su vez, se traduce en actitudes agresivas.

Uno de los factores, que al parecer influye con mayor fuerza y que da pie a actos violentos en niños, niñas y jóvenes, es la falta de diálogo. Al interior de las familias no existen canales adecuados de comunicación, llevando esto a que se presente desconfianza de hijos a padres.

Los programas de televisión han sido un referente importante para niños, niñas y jóvenes, ellos de identifican con algunos protagonistas en especial aquellos que representan; autoridad, poder, belleza; roles que han llevado a su cotidianidad elevando el índice de prostitución, vandalismo, alcoholismo, entre otros factores que desdibujan los valores y normas dentro de una sociedad.

Una evidencia es el siguiente gráfico, que expresa el informe de la entrevista realizada a los docentes, en la columna vertical se encuentra las opciones que corresponden a la pregunta iCuándo los(as) adolescentes se comportan de manera agresiva? ¿A cuál de los siguientes factores lo atribuyes?, y en la columna numerada la cantidad de respuestas dadas a esta interrogante por cada uno de ellos.

Tabla 2

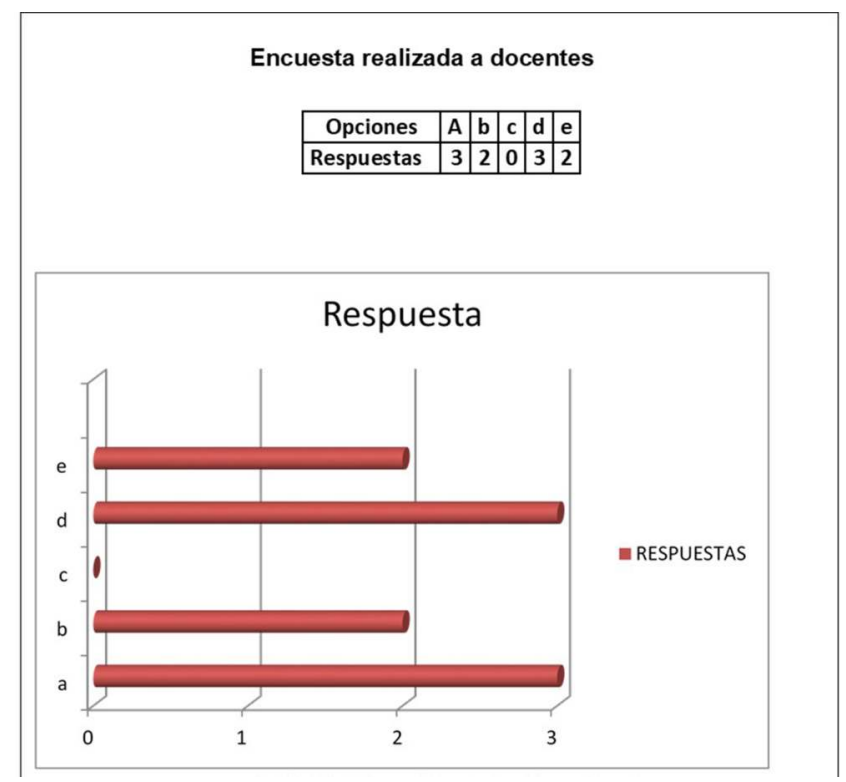

Elaboración de los investigadores 


\section{Encuesta realizada a estudiantes}

Los estudiantes como agentes primordiales en el proceso de enseñanza y aprendizaje, quienes a su vez son el eje de los centros educativos e instituciones educativas. En su proceso, ellos comparten, además de jornadas académicas, momentos lúdicos recreativos y deportivos.

Para el proyecto se tomarán como muestra diez (10) educandos, distribuidos entre el Centro Educativo Rural La Hermosa y la institución educativa Anorí.

En momentos de libertad, ellos se enfrentaron a una interrogante, en algunos casos solo se seleccionó una respuesta, pero en otros fueron varias las respuestas, que poco a poco avanza como material de insumo para el conocimiento de la agresividad y la forma óomo se evidencian los factores que la generan la agresividad en los niños y niñas.

Después de tabulada la entrevista (ver tabla 3) es evidente que la agresividad, según los estudiantes se presenta debido a:

- La han aprendido de otros, específicamente lo que día a día se refleja en su entorno familiar.

- La notoria inestabilidad emocional que viven las familias de los niños encuestados, hecho que conlleva a cada uno de estos niños a tornarse agresivos en situaciones que requieran convivencias.

- La influencia de los medios de comunicación como la televisión, ya que los niños toman como modelo las situaciones que se transmiten en estos medios.

De una forma más clara, se evidencia en el siguiente gráfico el informe de la entrevista realizada a los estudiantes: en la columna vertical se encuentra las opciones que corresponden a la pregunta ¿Cuándo los(as) adolescentes se comportan de manera agresiva? ¿A cuál de los siguientes factores lo atribuyes?, y en la columna numerada la cantidad de respuestas dadas a esta interrogante por cada uno de ellos. 
Tabla 3

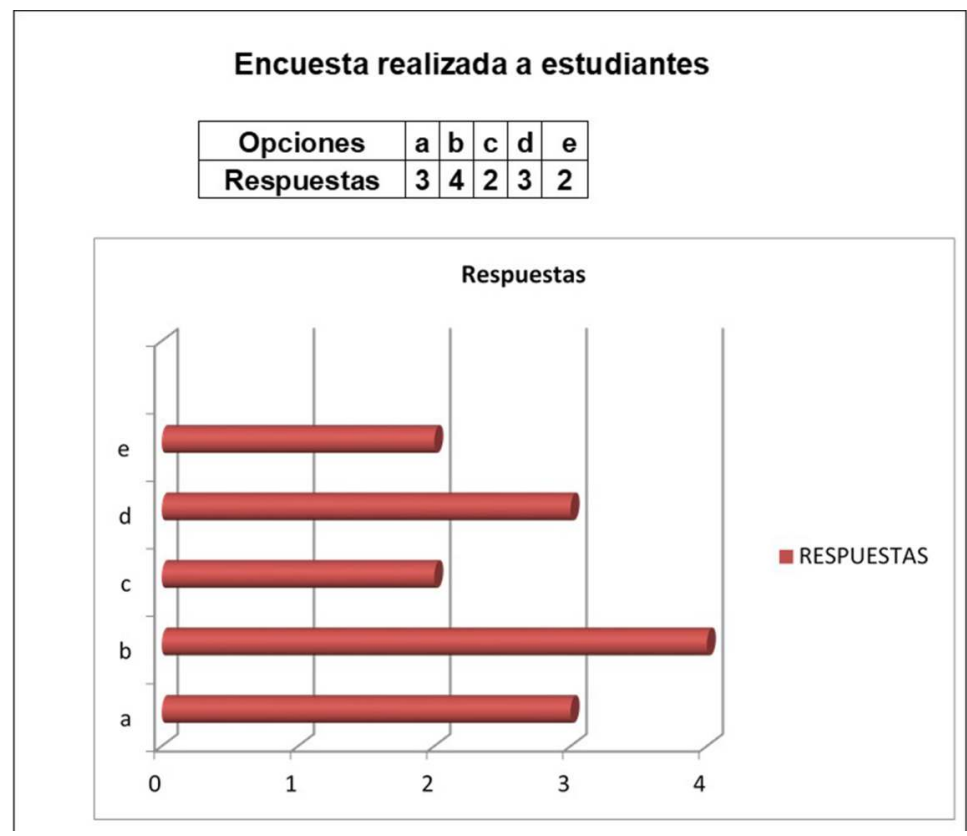

\section{Encuesta realizada a padres de familia}

Los padres de familia, como personas que están más cerca a las niñas y niños y tienen una gran responsabilidad en su formación, para el desarrollo de este proceso se aplicó la entrevistas a diez (10) de ellos, los cuales se desempeñan en diferentes ambientes, unos urbanos y otros rurales.

En momentos de libertad, ellos se enfrentaron a una interrogante, en algunos casos solo seleccionaron una respuesta, pero en otros fueron varias las respuestas, que se utilizó como material de insumo para el conocimiento de la agresividad y la forma cómo se evidencian los factores que la generan en los niños y niñas.

Después de tabulada la entrevista (ver tabla 4) es evidente que la agresividad según los padres de familia:

Se generaliza que en su hogar se viven situaciones de conflicto, ya que no se posee una buena relación y esta es fortalecida con los abusos físicos como una solución. 
Que los medios de comunicación como la televisión y los juegos electrónicos han influido de una forma transcendental en la agresividad de los niños y niñas, que toman como modelo lo que ven y oyen en las situaciones que se transmiten.

Que la agresividad de los niños también tiene su arraigo en la falta de afecto a los niños por parte de los padres, por parte de la familia mono - parentales.

Por otro lado, estos comportamientos en algunos casos, son producto lo que han vivido en la agresividad vivida en su entorno.

En menor grado se evidenció como causa de la agresividad en los niños, la educación dada en casa identificando la falta de diálogo como un factor determinante.

En el gráfico siguiente se muestran los resultados obtenidos en la entrevista aplicada a los padres de familia: en la columna vertical se encuentra las opciones que corresponden a la pregunta ¿Cuándo los(as) adolescentes se comportan de manera agresiva? ¿A cuál de los siguientes factores lo atribuyes?, Y en la columna numerada la cantidad de respuesta dada a este interrogante por cada uno de ellos.

Tabla 4

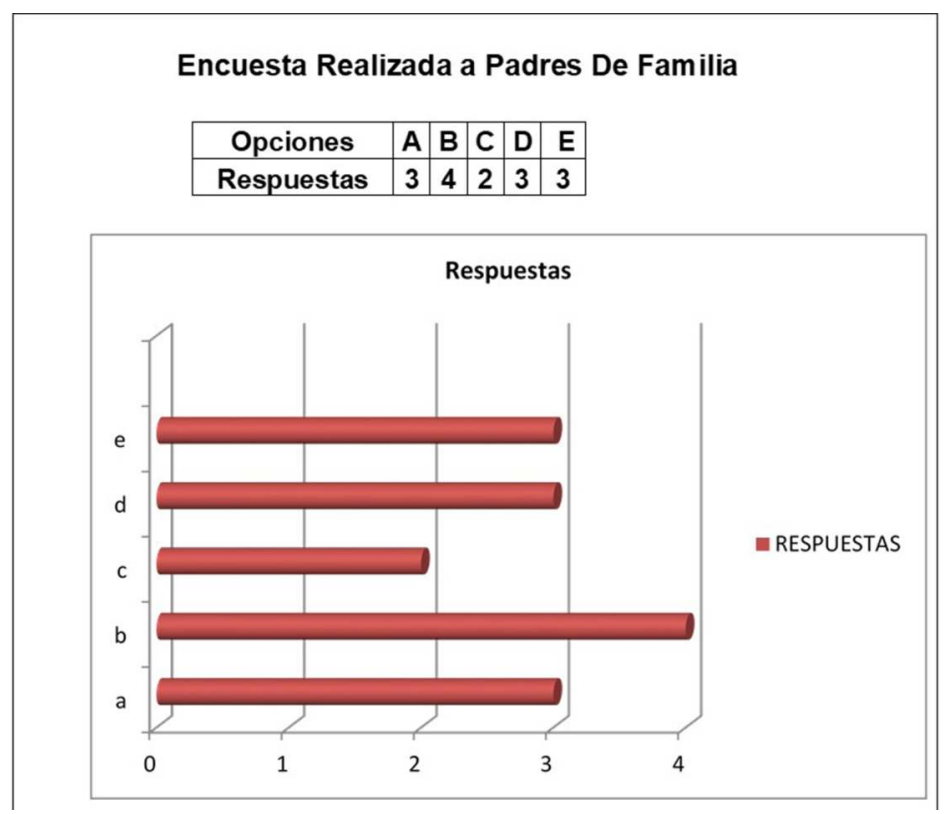




\section{RESULTADOS}

Los resultados de esta investigación confirman que la violencia escolar es el producto de una compleja combinación de variables, entre las que debe considerarse el entorno escolar, familiar y social. Los rasgos estereotipados, el conflicto intrafamiliar, la victimización escolar y, también, la tendencia hacia la agresión, la hostilidad y la ira de niñas, niños y jóvenes.

Los niños, niñas y jóvenes más agresivos suelen ser aquellos que padecen o han padecido durante la infancia separación de los padres (divorcio, nulidad matrimonial, desplazamiento forzado, desarraigo, abandono o muerte de uno de los progenitores); también aquellos que han sufrido malos tratos por parte de sus padres, otros familiares o personas con las que mantienen cierta dependencia (tutores, profesores, y otros).

El entorno escolar tiene una influencia decisiva en el comportamiento de los niños, niñas y jóvenes pues genera en ellos un sentimiento de competitividad y superioridad hacia el grupo de pares, haciéndolos recurrir a actitudes agresivas en el afán de demostrar quién es el o la mejor; pues ciertas condiciones proporcionan al niño señales de que su conducta agresiva puede tener consecuencias gratificantes.

Los niños y niñas que presentan conducta agresiva, por lo general habitan los sectores más violentos de su barrio y su estrato no es superior al dos, además se sienten identificados con los "líderes" de las pandillas del mismo sector.

A pesar de que la investigación es todavía incipiente y que se necesita un análisis más profundo de la relación entre la conducta agresiva y la construcción de las variables, los resultados encontrados deben llevarnos hacia una reflexión sobre los valores y conductas que, desde los procesos de socialización se trasmiten a nuestros estudiantes desde las Instituciones y centros educativos.

Es necesario incidir en el hecho de que los centros educativos no son lugares seguros ya que en ellos priman los conflictos que impiden el aprendizaje y el desarrollo de los alumnos. Sin embargo, es cierto que el contexto escolar no se escapa a la influencia de otros contextos sociales y en él se reproducen comportamientos que pueden empeorar el clima de convivencia y aprendizaje, como es el caso del acoso escolar.

En consecuencia, y puesto que las experiencias escolares son parte significativa de lo que los estudiantes aprenden de los otros, es necesario intervenir sobre 
aquello que representa una influencia negativa, sobre todo si se tiene en cuenta que los problemas de violencia entre iguales pueden ser la antesala de otras conductas problemáticas posteriores: abuso sexual, violencia en las relaciones de pareja o delincuencia.

Considerando lo anterior, cualquier medida preventiva o de intervención debe considerar, por un lado, cuáles son las circunstancias contextuales que rodean la situación y, por otro, cuál ha sido la influencia de los valores y actitudes vinculadas al interior de cada contexto.

\section{DISCUSIÓN}

En relación con las instituciones objeto de la investigación es importante resaltar que se involucran dos contextos con características diferentes pero que en esencia contemplan comportamientos similares: La zona urbana, en la que los niños cuentan con una serie de acciones que dejan en evidencia algunos factores determinantes para su comportamiento; y, la zona rural, un espacio un poco menos reducido para el desarrollo de la actividades, pero que en esencia también fueron determinantes para el desarrollo de la investigación.

Se requiere que, desde las aulas de clase, se establezca un espacio de concertación y mediación que apunten a una apropiación de mecanismo de concertación que ayuden a que la comunidad educativa (estudiantes, docentes y autoridades) tomen conciencia de su papel en el proceso de guía y formación de las personas a su cargo. Las aulas de clases deben propender por generar espacios de aprendizaje colaborativo y cooperativos en los que los niños se complementen y ayuden a sus pares.

También es importante tener en cuenta que las medidas preventivas y de intervención en las situaciones de violencia escolar, deben partir de la premisa de que los tipos de agresión, en su mayoría, poseen un carácter instrumental. La persona que las inicia busca un objetivo que, en principio, no tiene por qué ser el de dañar a la víctima, sino que ese daño puede ser un camino. 


\section{REFERENCIAS BIBLIOGRÁFICAS}

Comunidad educativa (2001). Institución Educativa Anorí, Manual de convivencia. Capítulo 6 y 7 Anorí: 2009-2011.70 pg.

Congreso de la República (2006). Ley de infancia y adolescencia. Ley 1098 recuperada de http://www.secretariasenado.gov.co/Senado/basedoc/ley_1098_2006.html

Constituyente, A. N. (1991). Constitución política de Colombia. Recuperado el 02 12, 2018.

Gadotti, M. (2002). Historia de las ideas pedagógicas. México: Siglo veintiuno.

Laplanche y Pontalis. "Diccionario del Psicoanálisis”, océanos, Barcelona, 1974. Pg. 720. Recuperado de https://documentop.com/laplanche-y-pontalis-diccionario-de-psicoanalisis 5a3ef2951723dd194d1bf02d.html

Manual de convivencia del Centro Edu cativo Rural La Hermosa. Capitulo. Medias faldas. 20082011.100p.

Morles, V. (1994). Planeamiento y análisis de investigaciones. El dorado Ediciones. Caracas. Venezuela.

Parra S. R. (1992). La escuela violenta. Tercer Mundo Editores. Bogotá. Pág. 312.

Pérez (2009). Definición de la agresividad infantil. www.psicologotarragona.com

Presidencia de la República (1993). Decreto 1423 regula las libertades.

Recuperado de https://diario-oficial.vlex.com.co/vid/decreto-54198538?_ga= 2.90520157.657168086.1562013834-1816350967.1562013834

Serrano, I (s.f). Agresividad Infantil, Ed. Pirámide (Citada En Internet Por La Psicóloga Gloria Marcellach Umbert). 